University of New Orleans

ScholarWorks@UNO

$5-1982$

\title{
Brewster and pseudo-Brewster angles of uniaxial crystal surfaces and their use for determination of optical properties
}

M. Elshazly-Zaghloul

R. M.A. Azzam

University of New Orleans, razzam@uno.edu

Follow this and additional works at: https://scholarworks.uno.edu/ee_facpubs

Part of the Electrical and Electronics Commons, and the Physics Commons

\section{Recommended Citation}

M. Elshazly-Zaghloul and R. M. A. Azzam, "Brewster and pseudo-Brewster angles of uniaxial crystal surfaces and their use for determination of optical properties," J. Opt. Soc. Am. 72, 657-661 (1982)

This Article is brought to you for free and open access by the Department of Electrical Engineering at ScholarWorks@UNO. It has been accepted for inclusion in Electrical Engineering Faculty Publications by an authorized administrator of ScholarWorks@UNO. For more information, please contact scholarworks@uno.edu. 


\title{
Brewster and pseudo-Brewster angles of uniaxial crystal surfaces and their use for determination of optical properties
}

\author{
M. Elshazly-Zaghloul \\ Electrical Engineering Department, Faculty of Engineering, Cairo University, Cairo, Egypt
}

R. M. A. Azzam

Department of Electrical Engineering, University of New Orleans, Lakefront, New Orleans, Louisiana 70122

Received August 26, 1981

\begin{abstract}
Brewster and pseudo-Brewster angles are defined for surfaces of transparent and absorbing uniaxial crystals parallel and perpendicular to the optic axis. Two Brewster angles of a transparent uniaxial crystal surface parallel to the optic axis, measured when the optic axis is oriented perpendicular and parallel to the plane of incidence, readily determine the ordinary and extraordinary indices $N_{o}$ and $N_{e} . N_{o}$ and $N_{e}$ can also be obtained from two Brewster angles measured on a surface perpendicular to the optic axis in contact with two media of different refractive indices. Conditions for the existence of two Brewster angles are discussed. The complex $N_{o}$ and $N_{e}$ of an absorbing uniaxial crystal can be derived from pseudo-Brewster-angle and minimum-reflectance data obtained in two symmetrical orientations of a surface parallel to the optic axis. An approximate, but accurate, explicit inversion procedure is presented for this purpose.
\end{abstract}

\section{INTRODUCTION}

When $p$-polarized parallel light is incident upon the surface of an optically isotropic transparent medium, the reflected wave disappears at a specific angle of incidence, the wellknown Brewster angle ${ }^{1} \phi_{B}$. If the medium becomes absorbing, the intensity of the reflected wave reaches a nonzero minimum at the so-called pseudo-Brewster angle $\phi_{p B}$. In this paper we extend the definition of Brewster and pseudoBrewster angles to optically anisotropic surfaces, specifically surfaces of transparent and absorbing uniaxial crystals. We also discuss the use of such angles to determine the optical properties of the crystal.

\section{BREWSTER ANGLES OF A TRANSPARENT UNIAXIAL GRYSTAL IN THREE SYMMETRICAL ORIENTATIONS}

We consider two crystal surfaces, one parallel and the other perpendicular to the optic axis. For the surface parallel to the optic axis, we consider in this section the two orientations when the optic axis is parallel and perpendicular to the plane of incidence and defer to Section 3 the general orientation with the optic axis at an arbitrary angle $\omega$ from the plane of incidence. The three symmetrical orientations, which are shown in Fig. 1, are described as follows:

(1) For a surface parallel to the optic axis, oriented such that the optic axis is perpendicular to the plane of incidence, Fig. 1(a), the reflection coefficient of incident $p$-polarized light is given $b^{2}$

$$
\begin{aligned}
R_{p p} & =\frac{N_{o}^{2} \cos \phi-n J}{N_{o}^{2} \cos \phi+n J}, \\
J & =\left(N_{o}^{2}-n^{2} \sin ^{2} \phi\right)^{1 / 2},
\end{aligned}
$$

where $\phi$ is the angle of incidence, $n$ is the refractive index of the isotropic medium of incidence, and $N_{o}$ and $N_{e}$ are the ordinary and extraordinary refractive indices of the crystal. The Brewster angle $\phi_{B}$ is obtained by setting

$$
R_{p p}=0,
$$

which gives

$$
\tan \phi_{B}=N_{o} / n \text {. }
$$

(2) For a surface parallel to the optic axis, oriented such that the optic axis is parallel to the plane of incidence, $R_{p p}$ is given $b^{2}$

$$
R_{p p}=\frac{N_{o} N_{e} \cos \phi-n J}{N_{o} N_{e} \cos \phi+n J} .
$$

$R_{p p}=0$ gives a second Brewster angle $\phi_{B}{ }^{\prime}$ such that

$$
\sin ^{2} \phi_{B}^{\prime}=\frac{n^{2} N_{o}^{2}-N_{o}^{2} N_{e}^{2}}{n^{4}-N_{o}^{2} N_{e}^{2}}
$$

(3) For a surface perpendicular to the optic axis, ${ }^{2}$ the $p$-reflection coefficient is given by

$$
R_{p p}=\frac{N_{o}^{2} \cos \phi-n\left(N_{e}^{2}-n^{2} \sin ^{2} \phi\right)^{1 / 2}}{N_{o}^{2} \cos \phi+n\left(N_{e}^{2}-n^{2} \sin ^{2} \phi\right)^{1 / 2}} .
$$

$R_{p p}$ is zero at a third Brewster angle $\phi^{\prime \prime}{ }_{B}$ given by

$$
\sin ^{2} \phi^{\prime \prime}{ }_{B}=\frac{n^{2} N_{e}^{2}-N_{o}^{4}}{n^{4}-N_{o}^{4}} \text {. }
$$

In all three symmetrical orientations there is no $p \rightarrow s$ polarization conversion (the cross-reflection coefficient $R_{p s}=$ 0 ), and the reflected beam is entirely extinguished at the Brewster angle, with no residual $s$-polarized component. Conditions for the existence of the Brewster angles $\phi_{B}^{\prime}$ and $\phi^{\prime \prime}{ }_{B}$ are discussed in Section 5 . 
(a)

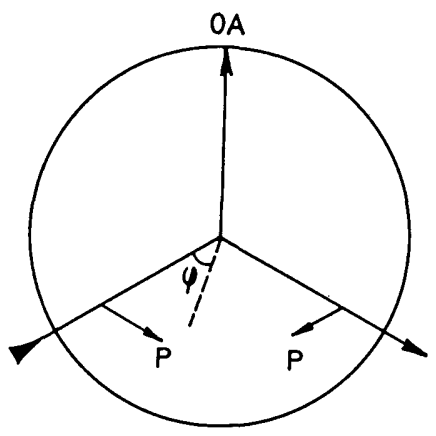

(b)

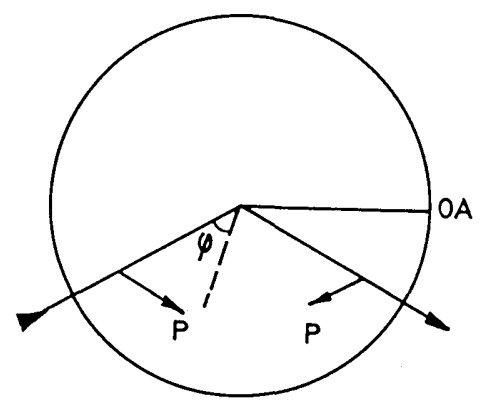

(c)

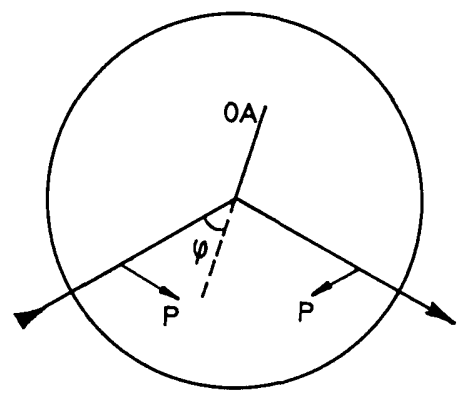

Fig. 1. Three symmetrical orientations of a uniaxial crystal with the optic axis $O A$ (a) parallel to the surface and perpendicular to the plane of incidence, (b) parallel to the surface and in the plane of incidence, and (c) perpendicular to the surface. When the incident light is $p$ polarized, the reflected light is likewise $p$ polarized.

\section{PSEUDO-BREWSTER ANGLE OF A} TRANSPARENT UNIAXIAL CRYSTAL WITH THE OPTIC AXIS PARALLEL TO THE

\section{SURFACE IN AN ARBITRARY ORIENTATION}

When $p$-polarized light is incident upon a uniaxial crystal surface parallel to the optic axis, with the optic axis inclined at an angle $\omega$ with respect to the plane of incidence, Fig. 2, the reflected light has $p$ - and $s$-polarization components that are determined by the following reflection coefficients ${ }^{3}$ :

$$
\begin{aligned}
R_{p p} & =\frac{A_{1} B_{4}+A_{2} B_{3}}{A_{1}+A_{2}}, \\
R_{p s} & =\frac{A_{1} A_{2}\left(B_{2}-B_{1}\right)}{\left(A_{1}+A_{2}\right)},
\end{aligned}
$$

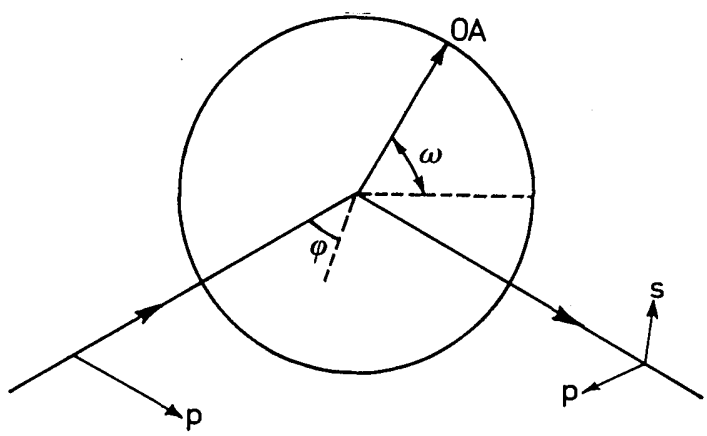

Fig. 2. Uniaxial crystal surface parallel to the optic axis $O A$, which makes an arbitrary angle $\omega$ with the plane of incidence. When the incident light is $p$ polarized, the reflected light has both $p$ - and $s$ polarized components.

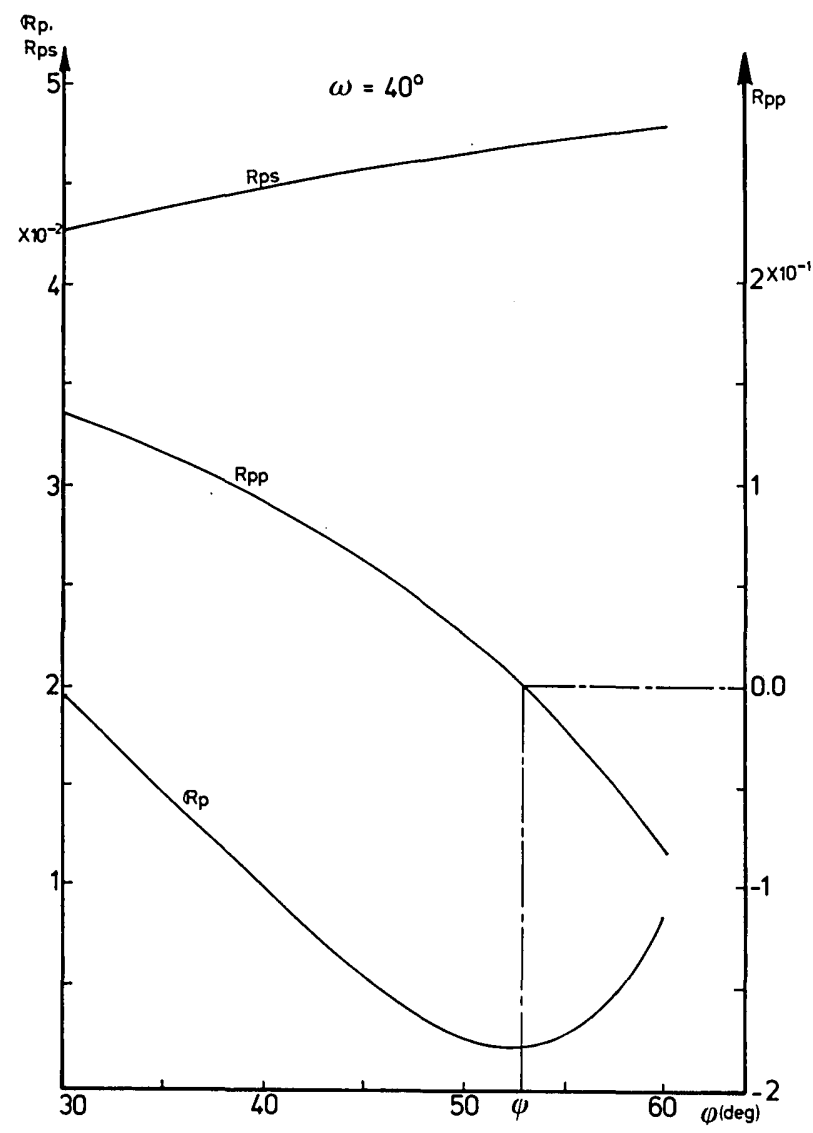

Fig. 3. The reflection coefficients $R_{p p}$ and $R_{p s}$ and the net $p$ reflectance $\mathcal{R}_{p p}$ versus the angle of incidence $\phi$ for a crystal surface of sodium nitrate parallel to the optic axis, which makes an angle $\omega=$ $40^{\circ}$ with the plane of incidence at wavelength $\lambda=5893 \AA$.

where

$A_{1}=J /\left[\left(n \sin ^{2} \phi+J \cos \phi\right) \tan \omega\right]$,

$A_{2}=N_{o} \tan \omega\left(I+n N_{o} \cos \phi\right) /\left(I N_{o} \cos \phi+n J^{2}\right)$,

$B_{1}=(n \cos \phi-J) /(n \cos \phi+J)$,

$B_{2}=\left(n N_{o} \cos \phi-I\right) /\left(n N_{o} \cos \phi+I\right)$,

$B_{3}=\left(N_{0}^{2} \cos \phi-n J\right) /\left(N_{o}^{2} \cos \phi+n J\right)$,

$B_{4}=\left(I N_{o} \cos \phi-n J^{2}\right) /\left(I N_{o} \cos \phi+n J^{2}\right)$,

$I=\left[N_{o}^{2} N_{e}^{2}-n^{2} \sin ^{2} \phi\left(N_{o}^{2} \sin ^{2} \omega+N_{e}^{2} \cos ^{2} \omega\right)\right]^{1 / 2}$, 
and $J$ is given by Eq. (2). The net parallel reflectance $R_{p}$ is given by

$$
\begin{aligned}
\mathscr{R}_{p} & =\left|R_{p p}\right|^{2}+\left|R_{p s}\right|^{2} \\
& =\mathscr{R}_{p p}+\mathscr{R}_{p s},
\end{aligned}
$$

where $\mathcal{R}_{p p}$ and $\mathscr{R}_{p s}$ are the diagonal $(p \rightarrow p)$ and cross $(p \rightarrow$ $s)$ reflectances, respectively.

We define two pseudo-Brewster angles for a given orientation $\omega$. At one pseudo-Brewster angle $\phi_{p B}(\omega)$ the net $p$ reflectance, Eq. (13), is minimum, and the reflected light has small residual $p$ - and $s$-polarized components. The other pseudo-Brewster angle $\phi_{p B}^{\prime}(\omega)$ is the angle of incidence of zero diagonal $p \rightarrow p$ reflectance. From Eq. (9) we see that $R_{p p}=$ 0 is satisfied when

$$
A_{1} / A_{2}=-B_{3} / B_{4},
$$

which can be solved for $\phi_{p B}^{\prime}$ as a function of $\omega, N_{o}$, and $N_{e}$. At the pseudo-Brewster angle $\phi_{p B}^{\prime}$ incident $p$-polarized light is totally converted to purely $s$-polarized light on reflection. The residual reflected intensity is determined by $\mathcal{R}_{p s}$.

Figure 3 shows the variation of the reflection coefficients $R_{p p}$ and $R_{p s}$ and the net $p$ reflectance $R_{p}$ with the angle of

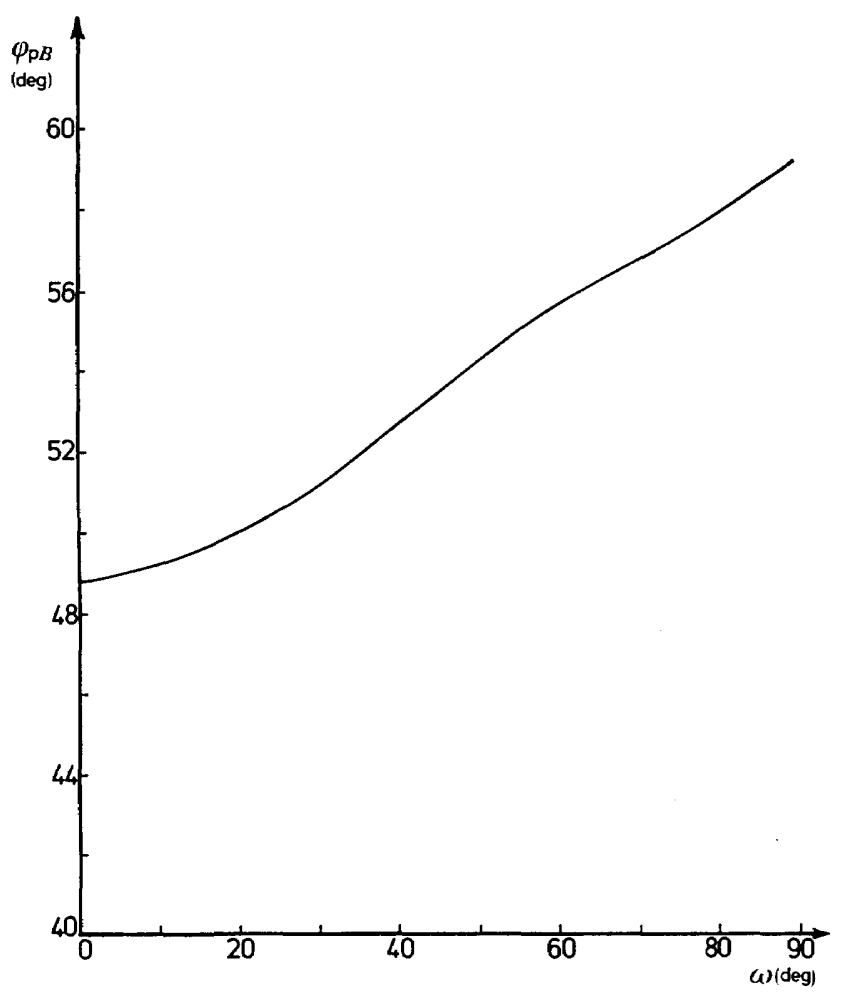

Fig. 4. The pseudo-Brewster angle $\phi_{p B}$ versus the orientation angle $\omega$ of the optic axis for a surface parallel to the optic axis of a sodium nitrate uniaxial crystal at $\lambda=5893 \AA$.

Table 1. Difference $\phi_{p B}-\phi_{p B}^{\prime}$ of the Two Pseudo-

Brewster Angles of a Uniaxial Crystal Surface of

Sodium Nitrate Parallel to the Optic Axis at Discrete Orientation Angles $\omega$ of the Optic Axis ${ }^{a}$

\begin{tabular}{lllllllll}
\hline$\omega$ & 0 & 10 & 20 & 30 & 50 & 70 & 80 & 90 \\
$(\mathrm{deg})$ & & & & & & & & \\
$\begin{array}{l}\phi_{p B}-\phi_{p B}^{\prime} \\
(\mathrm{deg})\end{array}$ & 0 & $\simeq 0$ & 0.02 & 0.04 & 0.06 & 0.04 & $\simeq 0$ & 0 \\
\hline
\end{tabular}

a $\lambda=5893 \AA$.

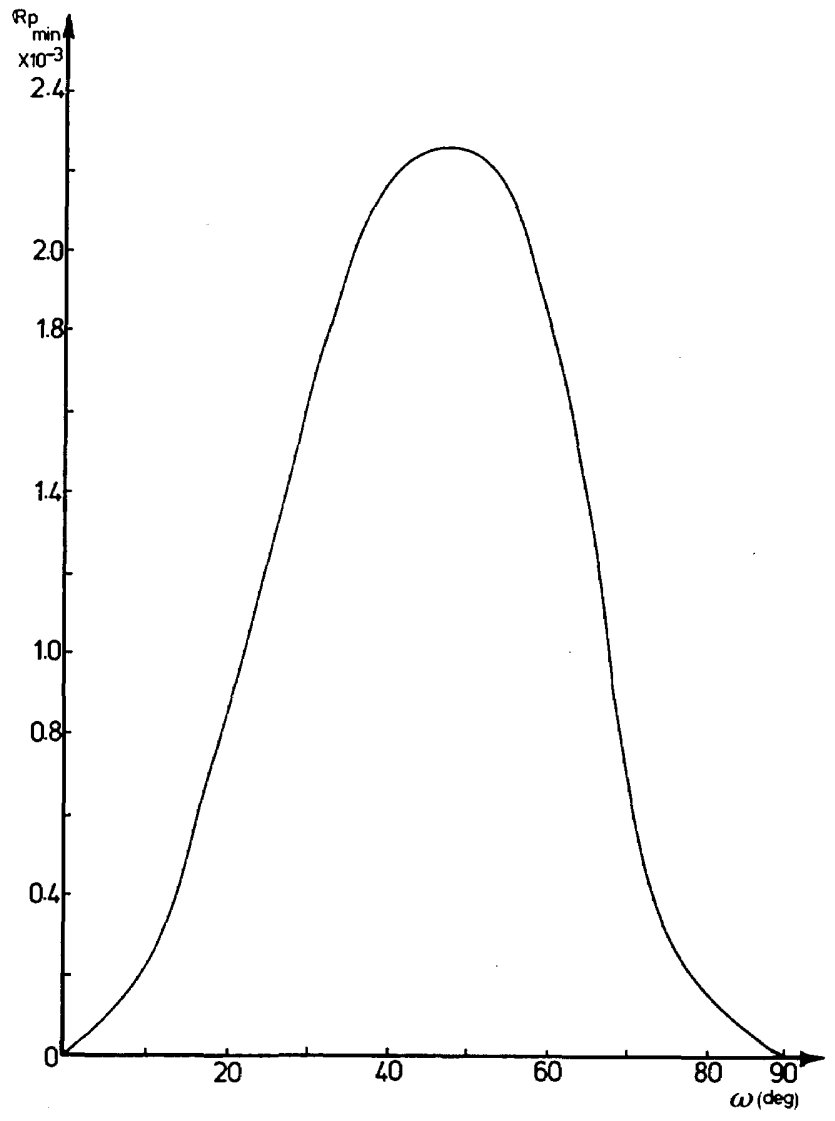

Fig. 5. The residual minimum reflectance $\mathcal{R}_{p_{\min }}$ as a function of $\omega$ for a surface parallel to the optic axis of a sodium nitrate uniaxial crystal at $\lambda=5893 \AA$.

incidence $\phi$ for a uniaxial crystal surface of sodium nitrate $\left(N_{o}\right.$ $=1.584, N_{e}=1.336$, at wavelength $\lambda=5893 \AA$ ) (Ref.4) parallel to the optic axis that makes $40^{\circ}$ with the plane of incidence. The angle of incidence of minimum net $p$ reflectance $\phi_{p B}$ is nearly the same as that at which $R_{p p}$ goes through zero, $\phi_{p B}^{\prime}$. Figure 4 shows the variation of the pseudo-Brewster angle $\phi_{p B}$ with the orientation angle $\omega$ of the optic axis for the sodium nitrate crystal. Figure 4 essentially gives also $\phi_{p B}^{\prime}$ as a function of $\omega$ because of the closeness of $\phi_{p B}$ and $\phi_{p B}^{\prime}$. The difference $\phi_{p B}-\phi_{p B}^{\prime}$ is given at discrete values of $\omega$ in Table 1. The maximum difference between these two pseudoBrewster angles is about $0.06^{\circ}$ and occurs at $\omega=45^{\circ}$. The two angles coincide for the symmetrical orientations $\omega=0$ and $90^{\circ}$. Figure 5 shows the residual minimum reflectance $\mathcal{R}_{p_{\min }}$ at $\phi_{p B}$, which nearly equals $\mathcal{R}_{p s}$ at $\phi_{p B}^{\prime}$, as a function of $\omega$. The minimum residual reflectance is maximum when $\omega=45^{\circ}$.

\section{PSEUDO-BREWSTER ANGLE OF AN ABSORBING UNIAXIAL CRYSTAL WITH THE OPTIC AXIS PARALLEL TO THE SURFACE}

For an absorbing uniaxial crystal, only pseudo-Brewster angles can be defined since the reflectance of $p$-polarized light never reaches zero even in symmetrical orientations. We restrict our attention to the case of a surface parallel to the optic axis and take gallium selenide $\left(N_{o}=3.7656-j 0.9062, N_{e}=2.7986\right.$ - j0.4687, at wavelength $\lambda=3000 \AA$ ) (Ref.5) as an example.

Figure 6 indicates a family of curves of the net $p$ reflectance $\mathcal{R}_{p}$ [computed by using Eqs. (9)-(12)] as a function of the 
angle of incidence $\phi$ at different orientation angles $\omega$ of the optic axis of a GaSe crystal. It is evident from Fig. 6 that the angle of incidence of minimum reflectance $\mathcal{R}_{p}$, the pseudoBrewster angle $\phi_{p B}$, shifts as the orientation angle of the optic axis $\omega$ changes. Figure 7 gives $\phi_{p B}$ as a function of $\omega$.

Figure 8 shows the variation [computed by using Eqs. (9)-(12)] of the real and imaginary parts of the complex $p \rightarrow$ $p$ and $p \rightarrow s$ reflection coefficients $R_{p p}$ and $R_{p s}$ with the angle of incidence $\phi$ for a fixed orientation of the optic axis $\omega=40^{\circ}$. Figure 8 also shows the curve of net $p$ reflectance $R_{p}$. Notice that the angle $(\phi)$ position of minimum $\mathcal{R}_{p}$ is nearly the same

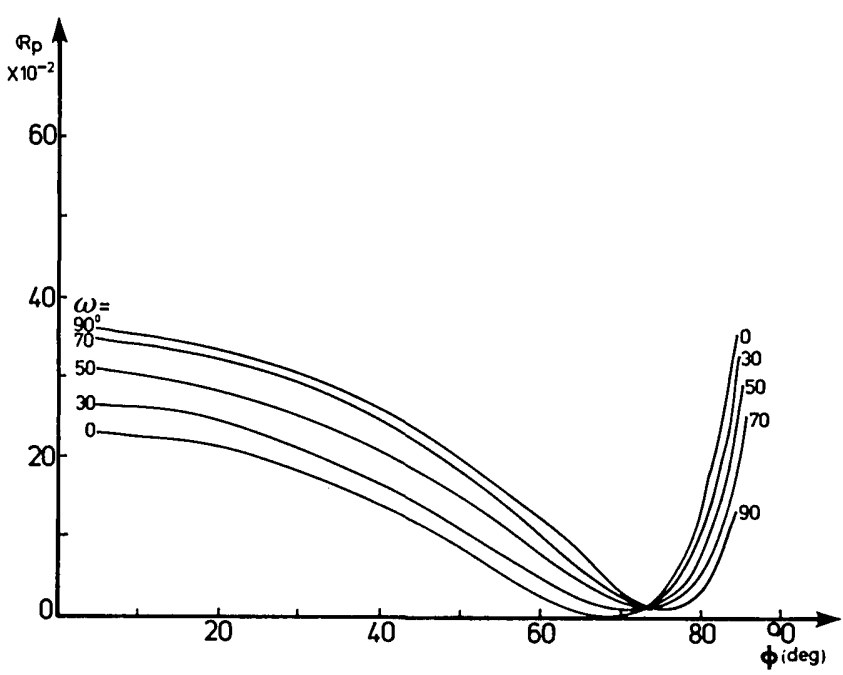

Fig. 6. The $p$ reflectance $\mathcal{R}_{p}$ as a function of angle of incidence $\phi$ at various orientations $\omega$ of the optic axis for a surface parallel to the optic axis of a GaSe crystal at $\lambda=3000 \AA$.

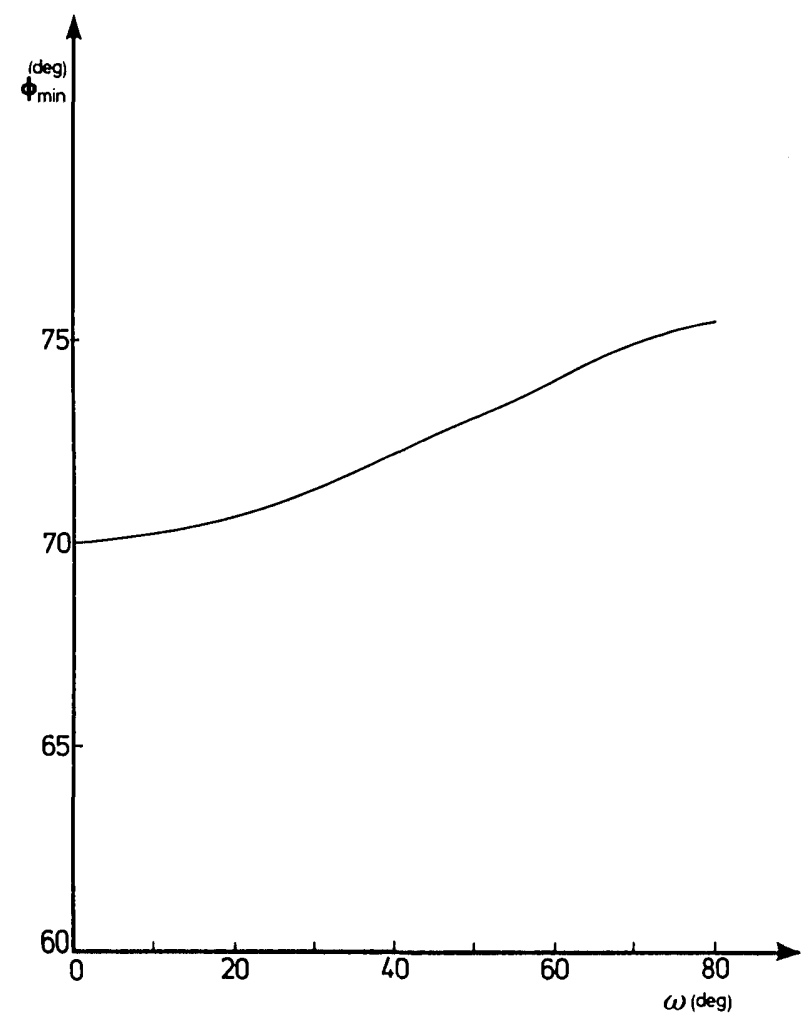

Fig. 7. The angle of minimum $p$ reflectance in Fig. 6, the pseudoBrewster angle $\phi_{p B}$, as a function of $\omega$.

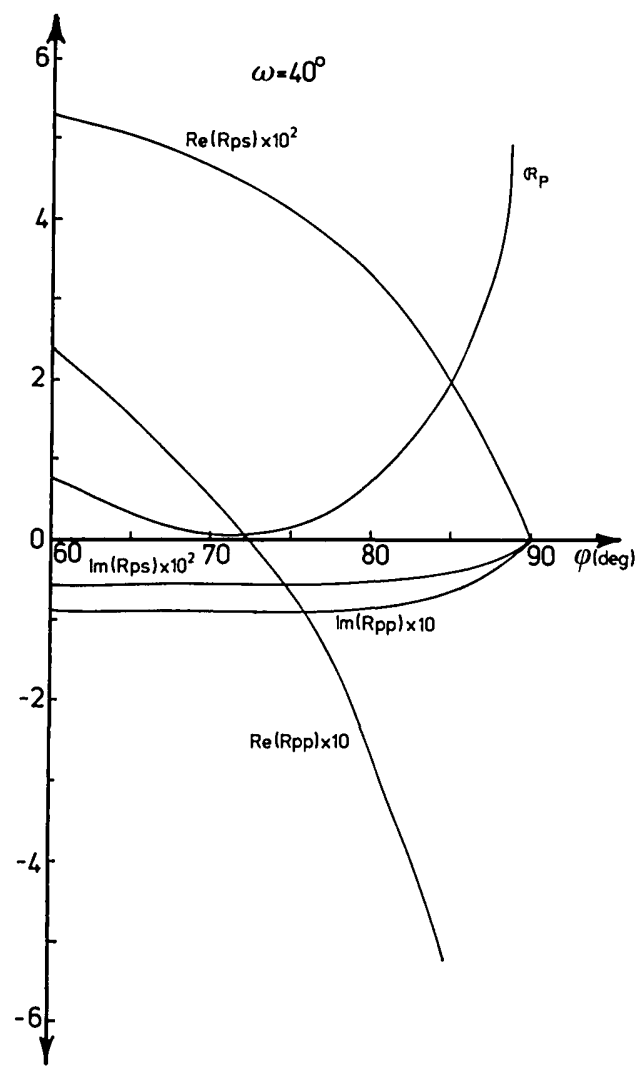

Fig. 8. The real and imaginary parts of the complex reflection coefficients $R_{p p}$ and $R_{p s}$, and the net $p$ reflectance $\mathcal{R}_{p}$ versus angle of incidence $\phi$ for a surface of GaSe crystal parallel to the optic axis, which is inclined by $\omega=40^{\circ}$ with respect to the plane of incidence; $\lambda=3000 \AA$.

as that at which the real part of $R_{p p}$ goes through zero. This conclusion, which is confirmed by numerical results and which remains valid as $\omega$ changes from 0 to $90^{\circ}$, will be used in Section 5 as the basis of an approximate method to determine the optical properties $N_{o}$ and $N_{e}$.

\section{DETERMINATION OF THE ORDINARY AND EXTRAORDINARY REFRACTIVE INDICES OF A UNIAXIAL CRYSTAL FROM BREWSTER AND PSEUDO-BREWSTER ANGLES}

\section{Transparent Crystals}

We describe two methods that involve measurement of two Brewster angles of one crystal surface. For a surface parallel to the optic axis, the Brewster angles $\phi_{B}$ and $\phi_{B}^{\prime}$ are measured for the two symmetrical orientations of the optic axis perpendicular and parallel to the plane of incidence, respectively. From $\phi_{B}$, the ordinary refractive index $N_{o}$ is immediately derived by using Eq. (2). Subsequently $N_{e}$ is obtained from $\phi_{B}^{\prime}{ }_{B}$ and $N_{o}$ by using Eq. (6). We assume the same immersion medium of known refractive index $n$ (e.g., air with $n=1$ ).

The second method employs measurement of the Brewster angles $\phi^{\prime \prime}{ }_{B 1}$ and $\phi^{\prime \prime}{ }_{B 2}$ of a crystal face perpendicular to the optic axis and immersed in two media of different refractive indices $n$ and $n_{2}$. Use of Eq. (8) twice yields two linear algebraic equations,

$$
\begin{aligned}
& N_{o}^{4} \cos ^{2} \phi_{B 1}^{\prime \prime}-n_{1}{ }^{2} N_{e}{ }^{2}+n_{1}{ }^{4} \sin ^{2} \phi^{\prime \prime}{ }_{B 1}=0, \\
& N_{o}{ }^{4} \cos ^{2} \phi_{B 2}^{\prime \prime}-n_{2}{ }^{2} N_{e}{ }^{2}+n_{2}{ }^{4} \sin ^{2} \phi_{B 2}^{\prime \prime}=0,
\end{aligned}
$$


which can be solved for $N_{o}^{4}$ and $N_{e}^{2}$; hence $N_{o}$ and $N_{e}$ are determined.

In the foregoing methods we took for granted the existence of all three Brewster angles $\phi_{B}, \phi_{B}^{\prime}$, and $\phi^{\prime \prime}{ }_{B}$. While $\phi_{B}$ can always be found from Eq. (2), $\phi^{\prime}{ }_{B}$ and $\phi^{\prime \prime}{ }_{B}$ may not exist for certain combinations of refractive indices $n, N_{o}$, and $N_{e}$. To discuss this point further, we define the normalized refractive indices:

$$
\eta_{o}=N_{o} / n, \quad \eta_{e}=N_{e} / n \text {. }
$$

In terms of $\eta_{o}$ and $\eta_{e}$, Eqs. (6) and (8) become

$$
\begin{aligned}
\sin ^{2} \phi_{B}^{\prime} & =\frac{\eta_{o}^{2} \eta_{e}^{2}-\eta_{o}^{2}}{\eta_{o}^{2} \eta_{e}^{2}-1}, \\
\sin ^{2} \phi_{B}^{\prime \prime} & =\frac{\eta_{e}{ }^{2}-\eta_{o}{ }^{4}}{1-\eta_{o}{ }^{4}} .
\end{aligned}
$$

Because the squared sine of a real angle is confined between 0 and 1, Eqs. $\left(6^{\prime}\right)$ and $\left(8^{\prime}\right)$ indicate that $\eta_{o}$ and $\eta_{e}$ must be restricted to the hatched domains of the $\eta_{o}-\eta_{e}$ plane shown in Figs. 9(a) and 9(b) so that the Brewster angles $\phi_{B}^{\prime}$ and $\phi^{\prime \prime}{ }_{B}$ may exist (i.e., are real).

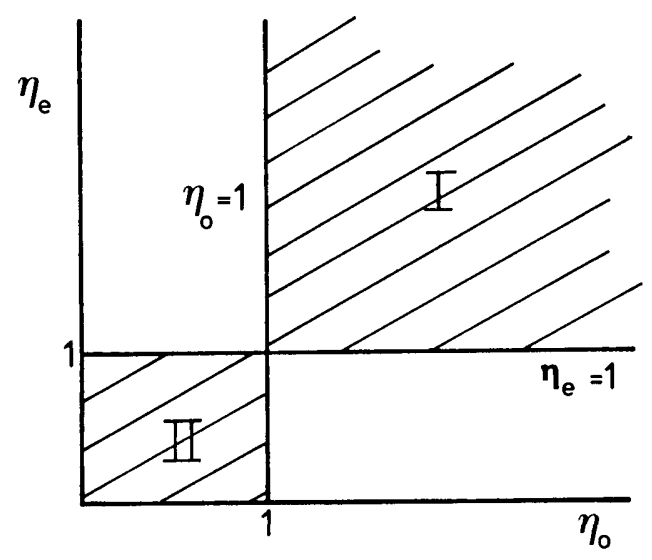

(a)

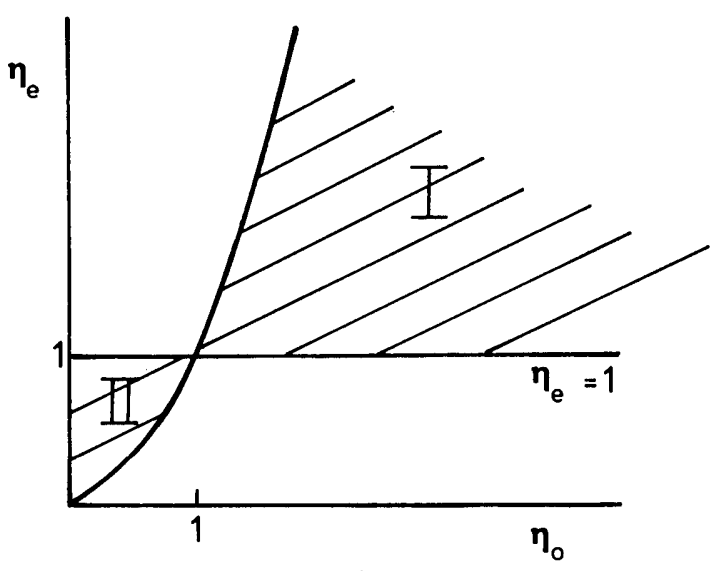

(b)

Fig. 9. Domains of existence (shown hatched) of the Brewster angles (a) $\phi_{B}^{\prime}$ and (b) $\phi^{\prime \prime}{ }_{B}$ in the plane of the normalized ordinary and extraordinary refractive indices $\eta_{o}$ and $\eta_{e}$. Subdomains I and II correspond to external and internal reflection, respectively.

\section{Absorbing Crystals}

For a surface parallel to the optic axis the pseudo-Brewster angle and associated minimum reflectance are measured for the two symmetrical orientations with the optic axis perpendicular $\left(\omega=90^{\circ}\right)$ and parallel $(\omega=0)$ to the plane of incidence. These are denoted by $\left(\phi_{p B}, \mathscr{R}_{p_{\text {min }}}\right)$ and $\left(\phi_{p B}^{\prime}, \mathscr{R}_{p_{\text {min }}}^{\prime}\right)$, respectively. The complex ordinary refractive index $N_{o}$ is derived from ( $\phi_{p B}, \mathcal{R}_{p_{\text {min }}}$ ) by making use of the condition that at $\phi_{p B}$, the real part of the complex reflection coefficient $R_{p p}$ is nearly zero, so that $R_{p p}$ is essentially pure imaginary, and we could write 6

$$
R_{p p}=-j \sqrt{\mathscr{R}_{p_{\text {min }}}}
$$

$N_{o}$ is then obtained by inverting Eq. (1); this gives

$$
N_{o}^{2}=\rho n^{2}\left[\rho \pm\left(\rho^{2}-\sin ^{2} 2 \phi_{p B}\right)^{1 / 2}\right] / 2 \cos ^{2} \phi_{p B},
$$

where

$$
\rho=\left(1+R_{p p}\right) /\left(1-R_{p p}\right) .
$$

$N_{e}$ is subsequently determined from $N_{o}$ and $\left(\phi_{p B}^{\prime}, \mathcal{R}_{p_{\min }}\right)$ by repeated use of the approximation $R_{p p}^{\prime}=-j \sqrt{R_{p_{\min }}^{\prime}}$ and inverting Eq. (3); this gives

$$
N_{e}=n \rho^{\prime} / N_{o} \cos \phi_{p B}^{\prime},
$$

where

$$
\rho^{\prime}=\left(1+R_{p p}^{\prime}\right) /\left(1-R_{p p}^{\prime}\right) .
$$

Simulated numerical calculations have verified the usefulness and accuracy of the previously described method of determining $N_{o}$ and $N_{e}$ of an absorbing uniaxial crystal from pseudo-Brewster-angle and minimum-reflectance data. For GaSe, with $N_{o}=n_{o}-j k_{o}=3.7656-j 0.9062, N_{e}=n_{e}-j k_{e}$ $=2.7986-j 0.4687$, the percentage errors of $n_{o}, k_{o}, n_{e}$, and $k_{e}$ by using this procedure are about $0.1 \%$ of each.

More accurate inversion of the $\left(\phi_{p B}, \mathcal{R}_{p_{\text {min }}}\right),\left(\phi_{p B}^{\prime}, \mathcal{R}_{p_{\text {min }}}^{\prime}\right)$ data to obtain $N_{o}$ and $N_{e}$ can always be attempted by using iteration and exact Eqs. (1) and (3), with $N_{o}$ and $N_{e}$ from Eqs. (18) and (20) serving as good initial values.

\section{ACKNOWLEDGMENTS}

This work was supported by the U.S. National Science Foundation, grant no. INT78-00373.

The results of this paper were presented at the 1979 Annual Meeting of the Optical Society of America in Rochester, New York [J. Opt. Soc. Am. 69, 1474 (A) (1979)].

\section{REFERENCES}

1. See, for example, M. Born and E. Wolf, Principles of Optics, 5th ed. (Pergamon, New York, 1975), p. 43.

2. See, for example, R. M. A. Azzam and N. M. Bashara, Ellipsometry and Polarized Light (North-Holland, Amsterdam, 1977).

3. T. P. Sosnowski, "Polarization mode filters for integrated optics," Opt. Commun. 4, 408-412 (1972).

4. J. Bennett and H. Bennett, "Polarization," in Handbook of Optics, W. G. Driscoll and W. Vaughan, eds. (McGraw-Hill, New York, 1978), Chap. 10.

5. F. Meyer, E. E. de Kluizenaar, and D. den Engelsen, "Ellipsometric determination of the optical anisotropy of gallium selenide," J. Opt. Soc. Am. 63, 529-552 (1973).

6. The minus sign in Eq. (17) is consistent with the $e^{j \omega t}$ time-dependence conventions. See R. H. Muller, "Definitions and conventions in ellipsometry," Surf. Sci. 16, 14-33 (1969). 